\title{
Commentary: Tolerance and Resistance of Pseudomonas aeruginosa Biofilms to Antimicrobial Agents-How P. aeruginosa Can Escape Antibiotics
}

\author{
Anaïs Soares ${ }^{1,2}$, François Caron ${ }^{1,3}$ and Manuel Etienne ${ }^{1,3 *}$ \\ 1 GRAM 2.0, EA 2656, Normandie Univ, UNIROUEN, Rouen, France, ${ }^{2}$ Microbiology Department, Rouen University Hospital, \\ Rouen, France, ${ }^{3}$ Infectious Diseases Department, Rouen University Hospital, Rouen, France
}

Keywords: Pseudomonas aeruginosa, biofilm, antibiotic failure, tolerance, resistance, phenotypic diversity, small colony variants

\section{A Commentary on}

OPEN ACCESS

Edited by:

Rustam Aminov,

University of Aberdeen,

United Kingdom

Reviewed by:

César de la Fuente,

Massachusetts Institute of

Technology, United States

*Correspondence:

Manuel Etienne

manuel.etienne@chu-rouen.fr

Specialty section:

This article was submitted to Antimicrobials, Resistance and

Chemotherapy,

a section of the journal

Frontiers in Microbiology

Received: 15 July 2019 Accepted: 03 September 2019 Published: 18 September 2019

Citation:

Soares A, Caron F and Etienne M (2019) Commentary: Tolerance and

Resistance of Pseudomonas aeruginosa Biofilms to Antimicrobial Agents-How P. aeruginosa Can

Escape Antibiotics.

Front. Microbiol. 10:2164. doi: 10.3389/fmicb.2019.02164
Tolerance and Resistance of Pseudomonas aeruginosa Biofilms to Antimicrobial Agents-How $P$. aeruginosa Can Escape Antibiotics

by Ciofu, O., and Tolker-Nielsen, T. (2019). Front. Microbiol. 10:913. doi: 10.3389/fmicb.2019.00913

In a recent article published in Frontiers in Microbiology, Ciofu and Tolker-Nielsen (2019), offered a comprehensive and didactic review on how Pseudomonas aeruginosa escapes antibiotic therapy in biofilms through tolerance and resistance mechanisms. Though their paper provided a wide panorama of the current knowledge in this field, we assume that the role of small colony variants (SCV) regarding antibiotic failure could have been discussed.

SCV are a phenotypic subset of the bacterial population surviving in biofilms. They have been described in chronic, persistent and recurrent biofilm-based infections, particularly in cystic fibrosis but also in chronic wound or device-related infections (Häussler et al., 2003; Proctor et al., 2006; Tielen et al., 2014; Johns et al., 2015). They were particularly well-described in Staphylococcus aureus (Proctor et al., 1995, 2006; von Eiff et al., 2006; Masoud-Landgraf et al., 2016), but were also identified in other species such as Pseudomonas aeruginosa (Häussler, 2004). P. aeruginosa SCV have a high biofilm-forming capacity and reduced swimming, swarming and twitching motilities (Déziel et al., 2001). They also have an increased expression of the psl and pel loci, encoding for Psl and Pel exopolysaccharides, that together with extracellular DNA form the biofilm matrix (Kirisits et al., 2005). Other studies reported that SCV emerged in biofilm cultures before any antibiotic exposure, and were not the sole bacterial population surviving antibiotic treatment (Déziel et al., 2001; Drenkard and Ausubel, 2002; Gloag et al., 2019). Because SCV constitute an adaptation of the parental strain to biofilm growth, contribute to biofilm production, and are encountered in situations where $P$. aeruginosa escapes antibiotics, their role in antibiotic failure through resistance mechanisms, tolerance mechanisms, or both can be questioned.

Recently, Balaban et al. (2019) recalled the definitions of antibiotic resistance and antibiotic tolerance in a consensus statement. Resistance is the ability of bacteria to replicate in the presence of antibiotic, whereas tolerance is the capability of a population to survive exposure to a bactericidal antibiotic without an increase in the MIC. The combination of both mechanisms in biofilms, has been named "recalcitrance" by Lebeaux et al. (2014). 
In specific situations, the biofilm growth might favor the selection of resistant mutants. As a fact, the exopolysaccharide matrix might reduce the diffusion in the deepest layers of the biofilm of some antibiotics such as aminoglycosides (Tseng et al., 2013). Bacterial cells entrapped into the matrix might hence be exposed to sub-inhibitory antibiotic concentrations, and resistant mutants might emerge (Kohanski et al., 2010; Jørgensen et al., 2013). In a study by Drenkard and Ausubel (2002), P. aeruginosa SCV with higher MICs toward aminoglycosides were recovered in sputum samples of cystic fibrosis patients treated with aminoglycoside-based antibiotic regimen. The mechanism of such increase in the MIC for SCV was not elucidated. As compared to the parent strain, the reduced susceptibility of SCV to aminoglycosides might result from increased interactions between positively charged aminoglycosides and negatively charged matrix components such as extracellular DNA (Chiang et al., 2013). But in this study as well as in others, the MICs increased only for aminoglycosides and not for fluoroquinolones or $\beta$-lactams (Singh et al., 2009; Wei et al., 2011).

However, the environmental conditions associated with biofilm growth (oxygen and nutrients limitations) mostly favor the expression of tolerance mechanisms (Walters et al., 2003; Borriello et al., 2004). Among the tolerant cells encountered in biofilms, persister cells are of particular interest (Lewis, 2010; Fauvart et al., 2011; Lebeaux et al., 2014). In various timekill assays in $P$. aeruginosa biofilm models, a biphasic killing curve, the hallmark of persistence, was observed after antibiotic exposure (Mulcahy et al., 2010; Benthall et al., 2015; RojoMolinero et al., 2016). Despite prolonged exposure at high antibiotic doses, viable, and culturable cells were still present in the biofilm while no resistant mutant was detected. Using high doses of antibiotic known to diffuse in the depth of the biofilm, such as ciprofloxacin (Walters et al., 2003; RodríguezMartínez et al., 2007), it is likely that antibiotic concentrations rapidly exceeded the mutation prevention concentration, so that pre-existing spontaneous resistant mutants might be eradicated. Regarding tolerance, the role of SCV in biofilm has not been specifically studied even for S. aureus. Singh et al. (2009), in a membrane-supported biofilm model, observed the emergence of S. aureus SCV among persister cells after treatment with ciprofloxacin, but not after treatment with amikacin, cefotaxime,

\section{REFERENCES}

Andersson, D. I., and Hughes, D. (2014). Microbiological effects of sublethal levels of antibiotics. Nat. Rev. Microbiol. 12, 465-478. doi: 10.1038/nrmicro3270

Balaban, N. Q., Helaine, S., Lewis, K., Ackermann, M., Aldridge, B., Andersson, D. I., et al. (2019). Definitions and guidelines for research on antibiotic persistence. Nat. Rev. Microbiol. 17, 441-448. doi: 10.1038/s41579-019-0196-3

Benthall, G., Touzel, R. E., Hind, C. K., Titball, R. W., Sutton, J. M., Thomas, R. J., et al. (2015). Evaluation of antibiotic efficacy against infections caused by planktonic or biofilm cultures of Pseudomonas aeruginosa and Klebsiella pneumoniae in Galleria mellonella. Int. J. Antimicrob. Agents 46, 538-545. doi: 10.1016/j.ijantimicag.2015.07.014

Borriello, G., Werner, E., Roe, F., Kim, A. M., Ehrlich, G. D., and Stewart, P. S. (2004). Oxygen limitation contributes to antibiotic tolerance of Pseudomonas oxacillin, or vancomycin. To what extent SCV contribute to biofilm persistence remains unelucidated.

From this analysis of the literature, we assume, in accordance with Ciofu and Tolker-Nielsen (2019) that resistance and tolerance mechanisms can both be expressed in biofilm infections. Those two ways for $P$. aeruginosa to escape antibiotics depend on different triggers. Sub-inhibitory antibiotic concentrations and active cell division favor the selection of genetically divergent resistant mutants. Conversely, lethal antibiotic concentrations trigger tolerance mechanisms, through a phenotypic adaptation, toward what could be called the "last hope bacterial response for survival" (Fajardo and Martínez, 2008; Andersson and Hughes, 2014). This phenotypic adaptation combines stress response activation depending on starvation strategies such as stringent response, SOS response or other metabolic pathways (Harms et al., 2016). SCV are part of these antibiotic-recalcitrant biofilm and for sure have specific metabolic characteristics, the increased exopolysaccharide matrix production being at the forefront (Harmsen et al., 2010; Moradali et al., 2017). Whether SCV behave differently than their parental strain regarding resistant mutants or persister cells emergence can to date not be ensured and needs further investigation. However, SCV might play a key role in the expression of persistence mechanisms, through their part in the biofilm structure, and may largely contribute to the environmental conditions associated with antibiotic recalcitrance.

In a daily practice, $P$. aeruginosa SCV can be detected on plate cultures. In most cases, they attest the presence of biofilm-related infection. SCV testing with usual antimicrobial susceptibility testing methods is also feasible, but little difference is expected regarding antimicrobial susceptibility between SCV and other bacterial populations entrapped in the biofilm. Nevertheless, informing clinicians of the presence of SCV seems crucial, as a signal that conditions are gathered for $P$. aeruginosa to escape antibiotics.

\section{AUTHOR CONTRIBUTIONS}

FC suggested the general commentary and reviewed the manuscript. AS and ME reviewed the literature and wrote the manuscript. All authors listed approved the work for publication. aeruginosa in biofilms. Antimicrob. Agents Chemother. 48, 2659-2664. doi: 10.1128/AAC.48.7.2659-2664.2004

Chiang, W.-C., Nilsson, M., Jensen, P. Ø., Høiby, N., Nielsen, T. E., Givskov, M., et al. (2013). Extracellular DNA shields against aminoglycosides in Pseudomonas aeruginosa biofilms. Antimicrob. Agents Chemother. 57, 2352-2361. doi: 10.1128/AAC.00001-13

Ciofu, O., and Tolker-Nielsen, T. (2019). Tolerance and resistance of Pseudomonas aeruginosa biofilms to antimicrobial agents-how $P$. aeruginosa can escape antibiotics. Front. Microbiol. 10:913. doi: 10.3389/fmicb.2019.00913

Déziel, E., Comeau, Y., and Villemur, R. (2001). Initiation of biofilm formation by Pseudomonas aeruginosa 57RP correlates with emergence of hyperpiliated and highly adherent phenotypic variants deficient in swimming, swarming, and twitching motilities. J. Bacteriol. 183, 1195-1204. doi: 10.1128/JB.183.4.1195-1204.2001 
Drenkard, E., and Ausubel, F. M. (2002). Pseudomonas biofilm formation and antibiotic resistance are linked to phenotypic variation. Nature 416, 740-743. doi: $10.1038 / 416740$ a

Fajardo, A., and Martínez, J. L. (2008). Antibiotics as signals that trigger specific bacterial responses. Curr. Opin. Microbiol. 11, 161-167. doi: 10.1016/j.mib.2008.02.006

Fauvart, M., De Groote, V. N., and Michiels, J. (2011). Role of persister cells in chronic infections: clinical relevance and perspectives on anti-persister therapies. J. Med. Microbiol. 60, 699-709. doi: 10.1099/jmm.0.030932-0

Gloag, E. S., Marshall, C. W., Snyder, D., Lewin, G. R., Harris, J. S., SantosLopez, A., et al. (2019). Pseudomonas aeruginosa interstrain dynamics and selection of hyperbiofilm mutants during a chronic infection. Mbio 10:e169819. doi: $10.1128 / \mathrm{mBio} .01698-19$

Harms, A., Maisonneuve, E., and Gerdes, K. (2016). Mechanisms of bacterial persistence during stress and antibiotic exposure. Science 354:aaf4268. doi: 10.1126/science.aaf4268

Harmsen, M., Yang, L., Pamp, S. J., and Tolker-Nielsen, T. (2010). An update on Pseudomonas aeruginosa biofilm formation, tolerance, and dispersal. FEMS Immunol. Med. Microbiol. 59, 253-268. doi: 10.1111/j.1574-695X.2010.00690.x

Häussler, S. (2004). Biofilm formation by the small colony variant phenotype of Pseudomonas aeruginosa. Environ. Microbiol. 6, 546-551. doi: 10.1111/j.1462-2920.2004.00618.x

Häussler, S., Ziegler, I., Löttel, A., von Götz, F., Rohde, M., Wehmhöhner, D., et al. (2003). Highly adherent small-colony variants of Pseudomonas aeruginosa in cystic fibrosis lung infection. J. Med. Microbiol. 52, 295-301. doi: 10.1099/jmm.0.05069-0

Johns, B. E., Purdy, K. J., Tucker, N. P., and Maddocks, S. E. (2015). Phenotypic and genotypic characteristics of small colony variants and their role in chronic infection. Microbiol. Insights 8, 15-23. doi: 10.4137/MBI.S25800

Jørgensen, K. M., Wassermann, T., Jensen, P. Ø., Hengzuang, W., Molin, S., Høiby, N., et al. (2013). Sublethal ciprofloxacin treatment leads to rapid development of high-level ciprofloxacin resistance during long-term experimental evolution of Pseudomonas aeruginosa. Antimicrob. Agents Chemother. 57, 4215-4221. doi: 10.1128/AAC.00493-13

Kirisits, M. J., Prost, L., Starkey, M., and Parsek, M. R. (2005). Characterization of colony morphology variants isolated from Pseudomonas aeruginosa biofilms. Appl. Environ. Microbiol. 71, 4809-4821. doi: 10.1128/AEM.71.8.4809-4821.2005

Kohanski, M. A., DePristo, M. A., and Collins, J. J. (2010). Sublethal antibiotic treatment leads to multidrug resistance via radical-induced mutagenesis. Mol. Cell 37, 311-320. doi: 10.1016/j.molcel.2010.01.003

Lebeaux, D., Ghigo, J.-M., and Beloin, C. (2014). Biofilm-related infections: bridging the gap between clinical management and fundamental aspects of recalcitrance toward antibiotics. Microbiol. Mol. Biol. Rev. 78, 510-543. doi: 10.1128/MMBR.00013-14

Lewis, K. (2010). Persister cells. Annu. Rev. Microbiol. 64, 357-372. doi: 10.1146/annurev.micro.112408.134306

Masoud-Landgraf, L., Zarfel, G., Kaschnigg, T., Friedl, S., Feierl, G., Wagner-Eibel, U., et al. (2016). Analysis and characterization of Staphylococcus aureus small colony variants isolated from cystic fibrosis patients in Austria. Curr. Microbiol. 72, 606-611. doi: 10.1007/s00284-016-0994-Z

Moradali, M. F., Ghods, S., and Rehm, B. H. (2017). Pseudomonas aeruginosa lifestyle: a paradigm for adaptation, survival, and persistence. Front. Cell. Infect. Microbiol. 7:39. doi: 10.3389/fcimb.2017.00039

Mulcahy, L. R., Burns, J. L., Lory, S., and Lewis, K. (2010). Emergence of Pseudomonas aeruginosa strains producing high levels of persister cells in patients with cystic fibrosis. J. Bacteriol. 192, 6191-6199. doi: 10.1128/JB.01651-09

Proctor, R. A., van Langevelde, P., Kristjansson, M., Maslow, J. N., and Arbeit, R. D. (1995). Persistent and relapsing infections associated with small-colony variants of Staphylococcus aureus. Clin. Infect. Dis. Off. Publ. Infect. Dis. Soc. Am. 20, 95-102. doi: 10.1093/clinids/20.1.95

Proctor, R. A., von Eiff, C., Kahl, B. C., Becker, K., McNamara, P., Herrmann, M., et al. (2006). Small colony variants: a pathogenic form of bacteria that facilitates persistent and recurrent infections. Nat. Rev. Microbiol. 4, 295-305. doi: 10.1038/nrmicro1384

Rodríguez-Martínez, J. M., Ballesta, S., and Pascual, A. (2007). Activity and penetration of fosfomycin, ciprofloxacin, amoxicillin/clavulanic acid and cotrimoxazole in Escherichia coli and Pseudomonas aeruginosa biofilms. Int. J. Antimicrob. Agents 30, 366-368. doi: 10.1016/j.ijantimicag.2007.05.005

Rojo-Molinero, E., Maci,à, M. D., Rubio, R., Moy,à, B., Cabot, G., LópezCausap,é, C., et al. (2016). Sequential treatment of biofilms with aztreonam and tobramycin is a novel strategy for combating Pseudomonas aeruginosa chronic respiratory infections. Antimicrob. Agents Chemother. 60, 2912-2922. doi: 10.1128/AAC.00196-16

Singh, R., Ray, P., Das, A., and Sharma, M. (2009). Role of persisters and smallcolony variants in antibiotic resistance of planktonic and biofilm-associated Staphylococcus aureus: an in vitro study. J. Med. Microbiol. 58, 1067-1073. doi: 10.1099/jmm.0.009720-0

Tielen, P., Wibberg, D., Blom, J., Rosin, N., Meyer, A.-K., Bunk, B., et al. (2014). Genome sequence of the small-colony variant Pseudomonas aeruginosa MH27, isolated from a chronic urethral catheter infection. Genome Announc. 2:e0117413. doi: 10.1128/genomeA.01174-13

Tseng, B. S., Zhang, W., Harrison, J. J., Quach, T. P., Song, J. L., Penterman, J., et al. (2013). The extracellular matrix protects Pseudomonas aeruginosa biofilms by limiting the penetration of tobramycin: limited tobramycin penetration protects biofilms. Environ. Microbiol. :2865-2878. doi: 10.1111/1462-2920.12155

von Eiff, C., Peters, G., and Becker, K. (2006). The small colony variant (SCV) concept - the role of staphylococcal SCVs in persistent infections. Injury 37(Suppl. 2), S26-S33. doi: 10.1016/j.injury.2006.04.006

Walters, M. C., Roe, F., Bugnicourt, A., Franklin, M. J., and Stewart, P. S. (2003). Contributions of antibiotic penetration, oxygen limitation, and low metabolic activity to tolerance of Pseudomonas aeruginosa biofilms to ciprofloxacin and tobramycin. Antimicrob. Agents Chemother. 47, 317-323. doi: 10.1128/AAC.47.1.317-323.2003

Wei, Q., Tarighi, S., Dötsch, A., Häussler, S., Müsken, M., Wright, V. J., et al. (2011). Phenotypic and genome-wide analysis of an antibiotic-resistant small colony variant (SCV) of Pseudomonas aeruginosa. PLoS ONE 6:e29276. doi: 10.1371/journal.pone.0029276

Conflict of Interest Statement: The authors declare that the research was conducted in the absence of any commercial or financial relationships that could be construed as a potential conflict of interest.

Copyright (c) 2019 Soares, Caron and Etienne. This is an open-access article distributed under the terms of the Creative Commons Attribution License (CC BY). The use, distribution or reproduction in other forums is permitted, provided the original author(s) and the copyright owner(s) are credited and that the original publication in this journal is cited, in accordance with accepted academic practice. No use, distribution or reproduction is permitted which does not comply with these terms. 